\title{
Space Environment Information System for Mission Control Purposes - a Decision Support System based on an architecture for space weather services
}

\author{
I. Dorotovič $\check{\check{c}}^{1,2,3}$, M. Pantoquilho ${ }^{1}$, N. Viana ${ }^{1}$ \\ and J. Moura-Pires ${ }^{4}$ \\ ${ }^{1}$ UNINOVA, Caparica, Portugal email: $\{$ id, mp, nv $\} @$ uninova.pt \\ ${ }^{2}$ Observatório Astronómico, GAUC, Coimbra, Portugal \\ ${ }^{3}$ Slovak Central Observatory, Hurbanovo, Slovak Republic email: dorotovic@suh.sk \\ ${ }^{4}$ DI/CENTRIA - Departamento de Informática/Centro de Inteligência Artificial, Caparica, \\ Portugal email: jmp@di.fct.unl.pt
}

\begin{abstract}
The radiation environment encountered in space by satellites is extremely variable and depends mainly on the satellite position and space weather. Although models for the concerned processes are available, most of them only represent the average conditions and neglect the dynamics of the system. Accurate prediction of the radiation environment remains an unsolved problem. Space weather can cause manifold problems to spacecraft (S/C) components, such as degradation of sensors and solar arrays and changes in on-board memories by Single Event Upsets (SEU). The final effect is a degradation of the $\mathrm{S} / \mathrm{C}$ overall performance and in extreme cases complete unavailability of services. When certain alarm conditions are reached, risk avoidance procedures may be invoked, e.g. switching off high voltages/biases/filters etc. and transition to protected operating modes. Once the detectors are off, there is just a rough estimation of when the conditions are safe again. Better prediction of radiation conditions and more accurate information could greatly improve these operations. Therefore it is necessary to monitor and predict the space weather effects and improve the space weather services.

In this paper we present a system for correlation, monitoring and predicting of space weather data, which is being developed by UNINOVA (Institute for the Development of New Technologies, Caparica, Portugal) in the frame of the European Space Agency (ESA) project, Space Environment Information System for Mission Control Purposes (SEIS) project.

This decision support system (DSS) provides Flight Control Teams with useful Space Weather information (past, current and future) to increase the ability to protect $\mathrm{S} / \mathrm{C}$ components from hazardous events and therefore prolong the lifetime of satellites and their scientific return. The system design was inspired by traditional business oriented DSS, based on Data Warehousing storage techniques, which collect and integrate historical and real-time data from heterogeneous sources, e.g. Space Weather data providers, S/C Telemetry and Orbital data and historical SEU records. The services provided include exploration and correlation analysis of data by established OLAP (On Line Analytical Processing) techniques, and near real-time monitoring of S/C instruments susceptible to Space Weather conditions. The monitoring service will directly depend on a Knowledge Base (representation of the domain experts knowledge), allowing it to suggest appropriate recovery actions whenever possible S/C damaging conditions are identified. Additionally, the system will incorporate forecasting services of well-known and commonly used physical radiation models, as well as new prediction techniques based on nonlinear models such as Artificial Neural Networks. For more information, please refer to: http://www2.uninova.pt/ca3/en/project_SEIS.htm
\end{abstract}

I. D. is supported by FCT, Lisboa, Portugal under grant number SFRH/BPD/14628/2003. 


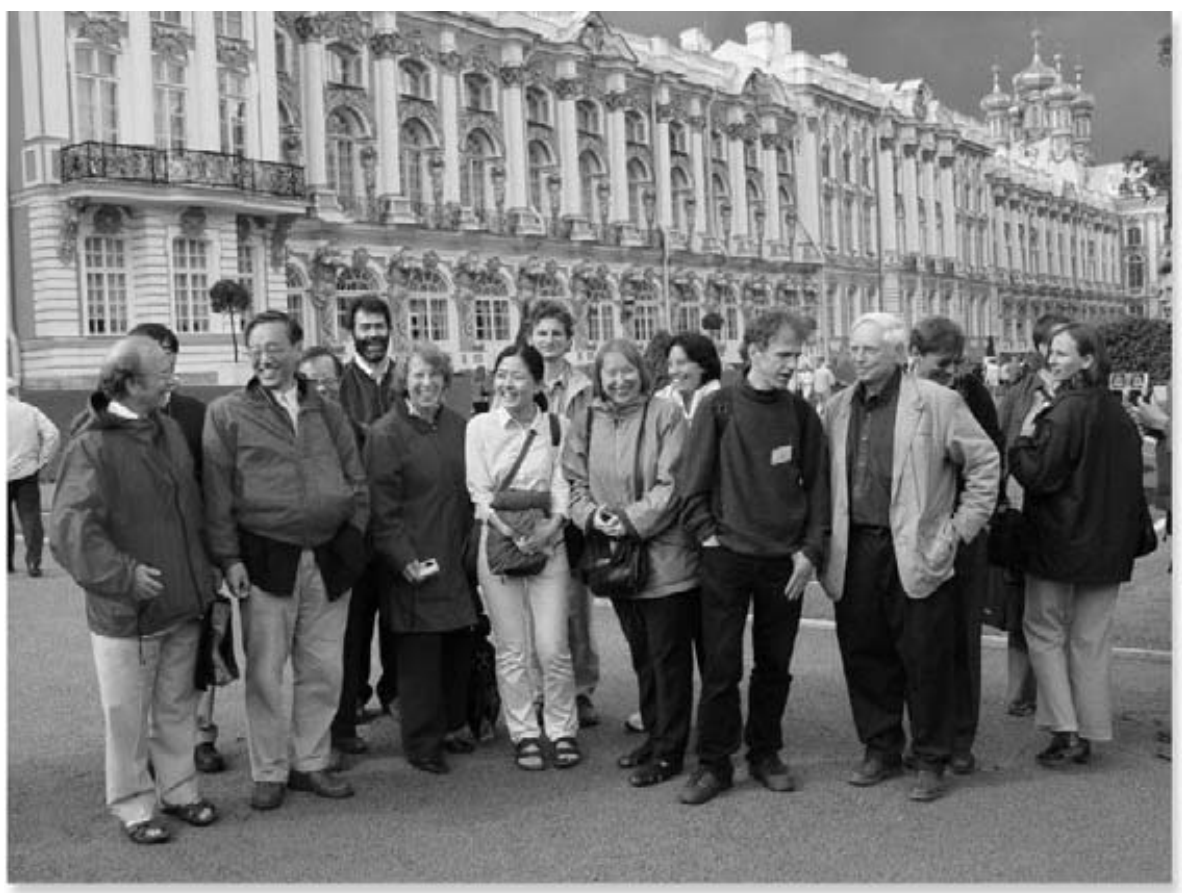

The Catherine's Palace

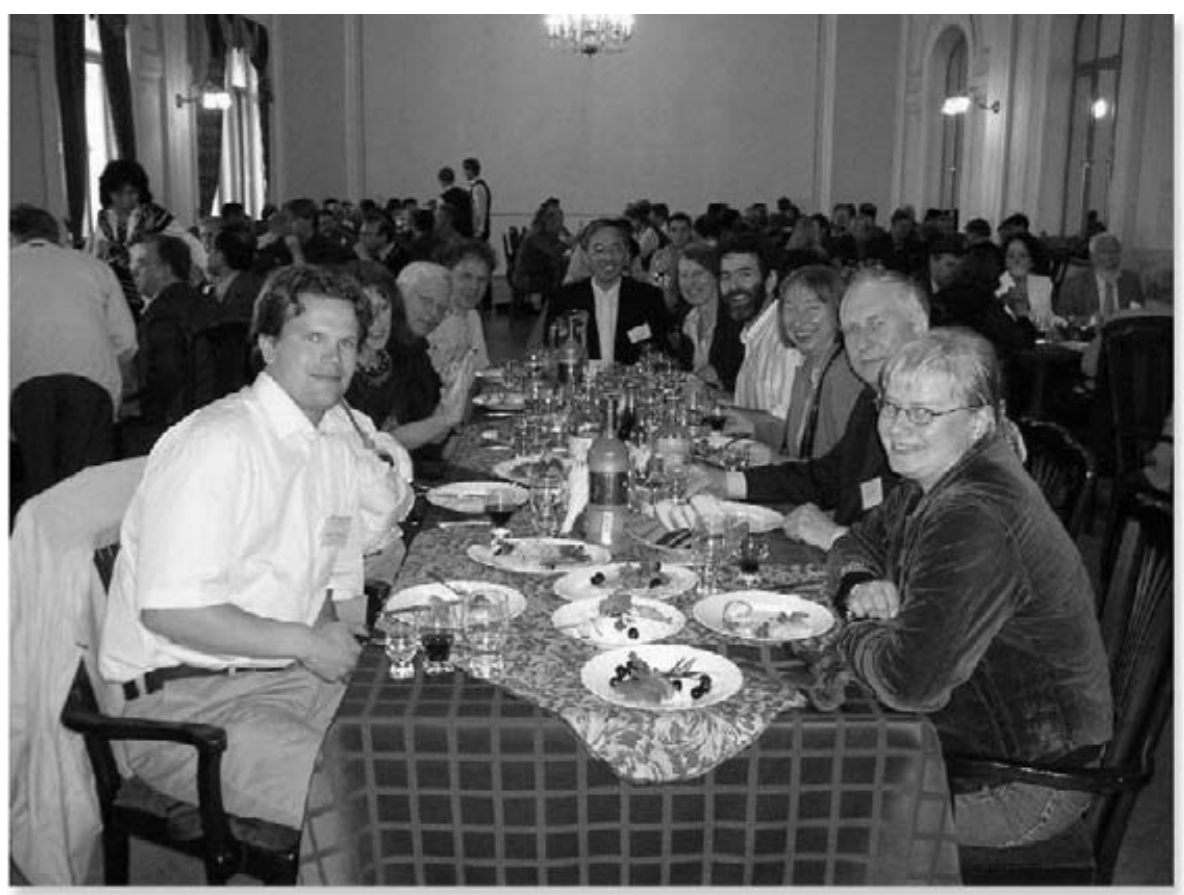

The Banquet 